\title{
PRESENTEEISM: AN INTRODUCTION TO A PREVAILING GLOBAL PHENOMENON
}

\author{
Maria Karanika-Murray \\ Department of Psychology, Nottingham Trent University \\ Cary L. Cooper \\ Manchester Business School, University of Manchester
}

Dr Maria Karanika-Murray, Department of Psychology, Nottingham Trent University, 50

Shakespeare Street, Nottingham, NG1 4FQ, UK, Email: maria.karanikamurray@ntu.ac.uk

Professor Sir Cary Cooper, Manchester Business School, University of Manchester, Booth Street East, Manchester M13 9SS, UK, Email: cary.cooper@manchester.ac.uk Author Note: Correspondence should be addressed to: Dr Maria Karanika-Murray 
Maria Karanika-Murray is Associate Professor in Occupational Health Psychology at Nottingham Trent University. Her research seeks to understand how workplaces that enable healthy and productive work can be developed, with a focus on workplace design, intervention evaluation, presenteeism, and older workers. Her work has been funded by the European Agency for Safety \& Health at Work, the UK Health \& Safety Executive, the UK Economic \& Social Research Council, Heart Research UK, and the European Commission.

Sir Cary L. Cooper, CBE... 


\section{PRESENTEEISM: AN INTRODUCTION TO A PREVAILING GLOBAL PHENOMENON}

In the current economic climate and the need to achieve more with less, many organizations strive to maintain productivity and remain competitive. This has a substantial impact on employee health, well-being, and work outcomes. One relatively recent phenomenon that is receiving increasing attention form a range of perspectives is presenteeism. Studies abound that not only show how prevalent presenteeism is across a range of occupations and sectors (Aronsson \& Gustafsson, 2005; Baker-McClearn, Greasley, Dale, \& Griffith, 2010; Biron, Brun, \& Ivers, 2006; Vézina et al., 2011) but also position it as more costly than absenteeism (CIPD, 2016). This cost can take many forms, including financial cost, performance and productivity, and individual health and well-being (Cooper \& Dewe, 2008; Kivimäki, Head, Ferrie, Hemingway, Shipley \& Vahtera, 2005; Stewart, Ricci, Chee, Morganstein \& Lipton, 2003b). This combination of high prevalence and high costs renders a comprehensive understanding of presenteeism and its damaging but also potentially beneficial outcomes necessary.

Because of its nature and impact presenteeism has attracted substantial research attention from a range of disciplines and perspectives, including work psychology, business and management, occupational health, public health, and economics. Research on presenteeism has exploded in the last few years, with a cursory search on Google Scholar of journal papers with 'presenteeism' in the title alone, yielded 236 publications in the last three years, 137 in the previous three years, and 72 in the three years before that, with the first studies emerging around 1996, when Cary Cooper (1996) first introduced the term.

This chapter offers an overview of current research and thinking on presenteeism.

Because of the scope and volume of this research, our overview will be necessarily selective, focusing on the major issues in the field. We draw from the rich and diverse perspectives and 
disciplines to explore definitions, theoretical models, antecedents, and outcomes of presenteeism, and extract research recommendations for future research.

\section{Definitional issues}

The term presenteeism is used to describe the phenomenon of people turning up at their jobs despite medical complaints and ill-health that would normally require rest and absence from work (Aronsson \& Gustafsson, 2005; Vingård, Alexanderson \& Norlund, 2004; Hemp, 2004; Johns, 2008). Cooper (1996) first defined presenteeism as a combination of physical presence but functional absence in the workplace: "being at work when you should be at home either because you are ill or because you are working such long hours that you are no longer effective" (p. 15). Johns (2010) offered a more concise definition of presenteeism as "showing up for work when one is ill".

A proliferation of definitions have been offered which reflect two main perspectives on presenteeism (Johns, 2010). European scholars tend to focus on presenteeism as the behaviour of attending work when one is sick as an outcome of job and occupational factors, whereas US scholars tend to be more concerned about productivity loss due to health problems (Schultz \& Edington, 2007; Burton et al., 2004). The European perspective defines presenteeism as "the phenomenon of people, despite complaints and ill health that should prompt rest and absence from work, still turning up at their jobs" (Aronsson, Gustafsson \& Dallner, 2000, p. 503; also see Dew, Keefe \& Small, 2005; and Johansson \& Lundberg, 2004). Juxtaposed to that, a definition offered by the American College of Occupational and Environmental Medicine is that of presenteeism as "the measurable extent to which health symptoms, conditions and diseases adversely affect the productivity of individuals who choose to remain at work" (Chapman, 2005, p. 2). The different approaches can be understood by looking at broader societal and economic differences. For example, health care system in the USA places more weight on private health insurance, whereas in Europe there 
has been a historical emphasis on social care with the government providing health insurance and a focus on wellness and rehabilitation into work (e.g., Ridic, Gleason \& Ridic, 2012).

The range of definitions also reflects the range of disciplinary perspectives and research and practice priorities. As Ashby and Mahdon (2010) note, "it is important to highlight that the concept of presenteeism has been understood in different ways. Presenteeism is not always associated with attending work when sick. Instead, it has also been used to describe those who are not unwell, but who come to work and seemingly intentionally or through disengagement do not perform to their best” (p. 13). A third perspective to the two outlined above views presenteeism as "the tendency to stay at work beyond the time needed for effective performance of the job" (Simpson, 1998, S.38), which is conceptually close to job insecurity.

Consensus is now emerging of presenteeism as attending work when one is unwell. This emerging consensus is important for three reasons. First, defining the behaviour of presenteeism (attending work when sick) in terms of its outcomes or consequences (performance loss) risks conflating cause and effect (Johns, 2010; Karanika-Murray \& Biron, in preparation). An association is not causation, and a relationship between poor health and productivity loss does not imply that poor health causes productivity loss; it is possible that third factor or factors can explain this association. Such definitional ambiguities are problematic in terms of measuring productivity loss, as in most cases it is difficult to know exactly when work is not getting done and there are numerous reasons for lost productivity which cannot be attributed to health.

Second, although different perspectives can offer rich and complementary understandings in an emerging field, they also determine the research questions and possible solutions prescribed. The risk is that without regular integration of knowledge and consensus 
building, this process may lead to the field splitting. It is unclear, for example, how findings from studies that use different building blocks (definitions and measures) can be integrated.

Third, definitional consensus is important for rigorous measurement tools. Existing measures focus on the frequency of presenteeism or job productivity and also range from a single item to longer scales. A popular self-report single-item measure by Aronsson \& Gustafsson (2005) asks respondents to indicate the frequency of attending work when ill within a recent time window (Hansen and Andersen, 2008; Johansson and Lundberg, 2004; Munir et al., 2009). Measures of presenteeism as the extent to which ill health interferes with job productivity (productivity loss) reflect a number of cognitive, emotional, and behavioural aspects of accomplishing work, with reference to being ill. Examples include the Work Limitations Questionnaire (WLQ), the Health and Work Performance Questionnaire (HPQ), the Stanford Presenteeism Scale (SPS-34 and SPS-13), the Work Productivity Short Inventory (WPSI), the Work and Health Interview (WHI), the Health and Labour Questionnaire (HLQ) and the Work Productivity and Activity Impairment Questionnaire (WPAI) (Schultz \& Edington, 2007). Most popular of these are the WLQ and HPQ, as both have strong validity and reliability and have been used in a variety of workplace settings, occupations, and health conditions (e.g., Leoppke Taitel, Haufle, Parry, Kessler \& Jinnett, 2009; Schultz \& Edington, 2007). Finally, objective assessment of productivity loss has also been attempted, such as using organisational records to assess the decrease in productivity associated with health problems in a sample of telephone customer-service employees (Burton, Conti, Chen, Schultz \& Edington, 1999). Although it is not our aim to review available measures of presenteeism (for a comprehensive review see Cyr \& Hagen, 2007; Schultz \& Edington, 2007), it is important to note that different definitions and approaches can lead to a range of measures of presenteeism and that inconsistent measurement is not favourable for integration of knowledge. 
Although pluralism in research is a useful and desirable way to kick start research on important topics, it can also inhibit integration of knowledge. In practical terms, agreeing a common language is essential for integrating current research and knowledge and developing solutions that can help employers and employees address presenteeism. In the case of presenteeism the risks of having too many diverse perspectives are even greater when we consider its substantial costs for individual health and performance and for organisational efficiency and productivity.

\section{Further issues: Understanding illness, attendance and related decisions}

The definition of presenteeism as 'showing up for work when one is ill' (Johns, 2010) raises some additional considerations: (1) what does illness mean, (2) what does attendance mean, and (3) by what criteria do individuals decide whether to attend work when they are ill? Could a deeper understanding of presenteeism by type of illness and type of attendance shed more light into what is acceptable or even sketch different types of presenteeism?

\section{Facets of illness}

The Oxford dictionary defines illness as 'a disease or period of sickness affecting the body or mind'. This intimates four dimensions of illness: an occurrence (a disease) of occasional or episodic illness, a temporal dimension (period of sickness) which may imply acuteness or chronicity, and two facets of illness (mental; physical). Johns (2010) distinguishes between episodic, acute and chronic conditions, whereas Gosselin and Lauzier (2010; in Gosselin, Lemyre \& Corneil, 2013) differentiate between occasional and chronic illness and between physical and psychological health. Garrow (2016) suggests that when considering the support that individuals (or their line managers) may need to manage presenteeism we should take into account the severity, duration, and frequency of a disease. In short, 'illness' is not a unidimensional construct. 
Understanding the nature of illness is essential for understanding its impact on work outcomes and the mechanisms through which presenteeism behaviour can lead to different work outcomes. For example, compared to psychological or mental ill-health, physical illhealth affects functional capacity, concentration or decision-making differently, and places different demands on the individual. Aligning the nature of the illness with the job tasks can help to identify (1) the work limitations that the individual is facing, (2) what the individual can do within these limitations and how he or she can remain involved in work, (3) whether presenteeism is an appropriate attendance behaviour, and (4) what support and resources they may need to continue to be at work or to facilitate return to work and recovery. In a recent study, Gosselin, Lemyre and Corneil (2013) found that "the specific nature of the illness has a marked impact on the decision process leading to either presenteeism or absenteeism" (p. 82). Specifically, they found that some health conditions, depending on their symptoms and how controllable they were, led to presenteeism whereas others, more debilitating conditions, led to absenteeism. Therefore, it is important to understand what illness means (or how it is perceived by the individual) and how it is linked to presenteeism behaviour.

\section{To attend or to absent}

The second consideration relates to attendance behaviour and specifically what type of response may be appropriate for different types of illness or the type or degree of debilitation from illness. At the two extremes, we have total absence or total presence during illness and regardless of the nature of the illness. In reality, complete absence is neither feasible not advisable. For a range of reasons (which we discuss later), individuals may decide to remain involved with work tasks during illness, i.e. engage in presenteeism behaviour. Of course, presenteeism work attendance in the face of illness may be more desirable than complete absence, as is implies some involvement with work, some less intensive or demanding tasks being completed, and some, albeit reduced, productivity. In 
reality, however, there is evidence that the costs and productivity loss associated with presenteeism is greater than that of absenteeism (Goetzel, Hawkins, Ozminkowski \& Wang, 2003; Hemp, 2004; Schultz \& Edington, 2007).

Despite commonality between the two behaviours, with the exception of very few conceptual papers, "absenteeism and presenteeism have developed along parallel paths without meeting despite being tied to a single decision" (Halbesleben, Whitman \& Crawford, 2014, p. 13). The alternative to the two extremes is well-managed attendance during illness as a way to facilitate gradual return to work. As Karanika-Murray and Biron (in preparation) note, whether a specific attendance behaviour is more appropriate than another is depends on the interaction between type of illness and job tasks. For example, if the illness affects functional capacity (e.g., broken leg) but the job tasks are mainly cognitive, do not require physical exertion, and can be performed as a flexible working from home option, then presenteeism can both help to achieve work tasks and support rehabilitation. If, on the other hand, the illness affects psychological or mental capacity and the nature of the work requires decision-making or social interaction with clients, then complete absence from work or temporary adjustment of work tasks would be advisable. In summary, and without venturing into a discussion on the management of presenteeism, the nature of the illness and nature of work tasks will require a different attendance response. Absenteeism vs. presenteeism are only two options that ought to be considered in light of the health condition and the work requirements.

\section{Voluntary vs. involuntary presenteeism}

The final consideration relates to the decision-making process that leads to presenteeism behaviour. Such an enacted decision is grounded in individuals' consideration of their current circumstances, including their health limitations and their work tasks but also broader contextual factors. As Garrow (2016) notes, “decisions on whether to 'present' or 
'absent' are, however, rarely based on simple health/task information. Other factors (both organisational and personal) come into play" (p. 2). At the individual level, not only people tend to ignore ill-health symptoms, ignore doctors' orders, and self-medicate (Kivimäki et al., 2005) but also even seemingly irrational or risky decisions may have adaptive purposes (Mellers, Schwartz \& Cooke, 1998). Individual action cannot be viewed in isolation from broader situational and contextual influences (Morgeson \& Hofmann, 1999) which is especially true in the case of presenteeism. Garrow (2016) also notes that "the relative dominance of these drivers is heavily context-dependent although evidence suggests that work factors tend to be more important" (p. 2). Therefore, it is important to understand how an individual's decision-making processes around being present or absent are influenced by the broader context.

Furthermore, how free are individuals to choose between presenteeism and absenteeism? For example, punitive attendance policies and procedures (Baker-McClearn et al., 2010), or line managers' misconception and misapplication of attendance and return to work procedures, or a workplace culture that encourages attending work at all costs (Dew et al., 2005) may foster presenteeism, even at the point where individuals substitute presenteeism for absenteeism (Caverley et al., 2007). From the lens of voluntary behaviour, the range of factors leading to presenteeism can be divided into 'voluntary' (e.g., work engagement) and 'involuntary' (e.g., attendance policies, job insecurity) and also suggest that involuntary influences are more frequent than voluntary influences (placing presenteeism cases linked to involuntary causes as high as 54\%; Biron, Brun \& Ivers, 2006). KaranikaMurray and Biron (in preparation) also support the distinction between voluntary and involuntary presenteeism whereas Baker-McClearn et al. (2010) define two types of presenteeism: institutionally-mediated presenteeism and personally-mediated presenteeism. Therefore, it is important to distinguish between presenteeism behaviour that is voluntary and 
based on free will, and presenteeism behaviour that an individual enacts because they feel pressured to attend work at the risk of their health.

\section{Conceptual work on presenteeism}

In contrast to empirical research, conceptual work on presenteeism that can helpfully explain the 'how' and 'why' of the phenomenon and that systematically integrates empirical research is sparse. In other words, the volume of research into presenteeism to-date is disproportionately large in comparison to the theorising offered. Two main groups of theories have been developed, one focusing on the decision process behind presenteeism behaviour and the other on the determinants of presenteeism.

The model of illness flexibility (Johansson \& Lundberg, 2004) suggests that attendance requirements (the negative consequences that employees face due to absence) and adjustment latitude (modifications in the workload of sick employees) are key determinants of both sickness attendance (presenteeism) and absence. Using survey data from 4924 people, they found partial support for the associations between adjustment latitude and attendance requirements with work behaviours, such that high attendance requirements increased the probability of attending work whilst ill (adjustment latitude was not associated with presenteeism). Aronsson and Gustafsson (2005) proposed that presenteeism is an outcome of a decision process on whether to go to work or not. They also suggested that there are two different types of attendance demands that influence sickness presenteeism: personally related factors such as one's financial situation and individual boundarylessness. Boundarylessness is linked to work factors such as control over pace of work, replaceability, sufficient resources, and time pressure and conflicting demands. Expanding on Aronsson and Gustafsson (2005), Hansen and Andersen (2008) outlined the impact of organizational and individual factors in the behavioural choice process. Johns's (2010) integrated model proposes that a health event triggers a choice between presenteeism and absenteeism. It also 
identifies the factors that influence this choice and consequently occurrence of absenteeism and presenteeism behaviour as the work context (e.g., ease of replacement, absence policy, or job demands) and individual factors (e.g., personality or work attitudes). It is important here to clarify the nature of this 'choice' since, as noted earlier, presenteeism can be voluntary or involuntary. As an extension of this conceptual work, Miraglia and Johns (2015) proposed a more elaborate dual-path model which views job attitudes and health as the mediators of the range of personal and work-related factors that lead to presenteeism or absenteeism.

These models concur on three fronts: (1) viewing presenteeism behaviour as a decision process, (2) acknowledging the relational dynamics between presenteeism and absenteeism, and (3) highlighting the interaction between illness, individual factors, and work-related factors as decision levers. The notion of adjustment latitude, whether implicit or explicit, is important as it can help to accommodate the needs of the individual vis-à-vis the requirements of the job and the nature of the illness. In this way, adjustment latitude can help to balance the range of pressures and determinants of presenteeism in order to support individual health and performance.

In terms of determinants of presenteeism, a range of typologies have been offered. Aronsson and Gustafsson (2005) described two types of attendance demands that influence presenteeism: personal factors (e.g., financial situation and individual boundarylessness) and work factors (e.g., control over pace of work, replaceability, sufficient resources, time pressure and conflicting demands). Biron and Saksvik (2009) organised the literature on determinants of presenteeism into work-related (e.g., difficulty in being replaced), dispositional (e.g., guilt and pressure factors), and situational determinants (e.g., financial insecurity). Baker-McClearn et al. (2010) summarised the workplace factors that influence an individual' $\mathrm{s}$ decision to either attend or be absent from work when ill as personal motivations (e.g., loyalty to own professional image) and workplace pressures (e.g., 
workplace culture). Similarly, Johns's (2010) dynamic integrated model suggests that the choice between presenteeism and absenteeism relies on evaluations of the work context (i.e., ease of replacement, absence policy, job demands, etc.) and individual factors (i.e., personality and work attitudes). Gosselin, Lemyre and Corneil (2013) proposed an integrated model of the determinants of presenteeism and absenteeism behaviour, incorporating the influence of health problems, demographic characteristics, individual factors, and organizational factors. Finally, pressure factors such as high personal or professional obligations to work, and motivational factors such as pleasure derived from work and job satisfaction have also been highlighted (Miraglia \& Johns, 2015).

Common in these typologies is the suggestion that there are multiple levels of determinants of presenteeism behaviour and that these operate synergistically rather than in isolation. It is also worth noting that "work-related factors seem to be slightly more important than personal circumstances or attitudes in determining people's 'decision' to go ill at work. However, the relatively low explanatory power of these combined factors suggests that there are still many unknowns in this field of research" (Hansen \& Andersen, 2008, p. 956).

Available conceptual work focuses predominantly on understanding the process by which a range of factors determine presenteeism behaviour, but very little theoretical attention has been invested in understanding the outcomes of presenteeism. Empirical work mirrors this as it has tended to focus on categorizing the determinants of presenteeism, essentially viewing it as static end-behaviour. As a result, we have little insight into the psychological mechanisms and psychological processes that drive presenteeism behaviour (Cooper \& Lu, 2016; Karanika-Murray, Pontes, Griffiths \& Biron, 2015) and its outcomes for individuals and organizations. Indeed, Vingård, Alexanderson and Norlund's (2004) review identified just eight studies on the consequences of sickness presenteeism for the employer but failed to identify any empirical evidence focusing on its consequences for the individual, 
concluding that " $[\mathrm{t}]$ he current body of scientific literature does not provide sufficient evidence to draw conclusions on the consequences of sickness presence" (p. 216). Their observation still holds today.

Considering how deleterious presenteeism can be to employee health (in terms of future ill-health, well-being, or sickness absence, to name a few) and costly to organizations (in terms of productivity loss, replacement costs, colleague morale, for example) and the importance of understanding its impact in order to develop ways to mitigate deleterious effects, this lack of conceptual attention on outcomes and the mechanisms that drive presenteeism is rather odd. There are two exceptions. Karanika-Murray et al. (2015) proposed that presenteeism is a combination of physical presence and psychological absence, tapping into the notion of presenteeism as working at reduced capacity. They offered empirical evidence that motivational states (work engagement and work addiction) fully mediate the relationship between presenteeism behaviour and job attitudes (job satisfaction), viewing presenteeism as a determinant rather than end-behaviour. In addition, Cooper and $\mathrm{Lu}$ (2016) proposed the social cognitive model of presenteeism as a plausible exploration of the possible psychosocial mechanisms that drive presenteeism behaviour. They outlined how self-efficacy and estimates of outcomes expectancy together impact on goal setting which, in turn, impacts on presenteeism behaviour and subsequently performance attainment. In the process, their model also considers the influence of both person and contextual variables. This is one of the few dynamic models of presenteeism that can help to understand how decisions to attend work while ill are made and that also view presenteeism as one link in a chain of effects.

Being overcrowded with a-theoretical studies with a singular focus on its prevalence, determinants, and impact (in monetary, health and performance terms), the field of presenteeism research is "markedly atheoretical" (Johns, 2010) and in need of theoretical development (Dickson, 2013). Investment in conceptual work and in understanding 
presenteeism as one part of a chain of effects is needed to organise the volume of empirical studies and allow a more comprehensive understanding of presenteeism.

\section{Empirical research on the antecedents of presenteeism}

As mentioned, the majority of empirical research on presenteeism has focused on its determinants, offering useable and testable theoretical models to explain the factors that drive presenteeism behaviour. Here we outline specific antecedents.

Financial pressures and job insecurity are among one of the most common reasons why people go to work despite being ill (Aronsson \& Gustafsson, 2005; Bierla, Huver \& Richard, 2013; Barnes, Buck, Williams, Webb \& Aylward, 2008; Bergstrom, Bodin, Caverley, Cunningham \& MacGregor, 2007; Hagberg, Lindh, Aronsson \& Josephson, 2009; Prater \& Smith, 2011; Widera, Chang \& Chen, 2010). However, it is possible that job insecurity may indicate other underlying factors associated with presenteeism including, for example, the norms and climate of the workplace. Depression has also been inked with overall work limitations and productivity loss (Burton, Pransky, Conti, Chin-Yu \& Edington, 2004); McTernan, Dollard \& LaMontagne, 2013). Job satisfaction is a strong predictor of the likelihood of attending work whilst ill (Aronsson \& Gustafsson, 2005; Caverley et al., 2007; Krohne \& Magnussen, 2011) even among those who experienced chronic nonspecific musculoskeletal pain (de Vries, Brouwer, Groothoff, Geertzen \& Reneman, 2011), although not all research has supported a positive link (Rosvold \& Bjertness 2001). Work engagement is also closely associated with presenteeism (Admasachew \& Dawson, 2011; KaranikaMurray et al., 2015). A number of job characteristics have been implicated in presenteeism, such as job control (Aronsson \& Gustafsson, 2005; Alavinia, Molenaar \& Burdorf, 2009; Gosselin et al., 2013). Biron, Brun, and Ivers (2006) however found that lack of control was a determinant of presenteeism but only for workers with a rather benign health issue (for those reporting $<9$ days of presenteeism). Increased time pressure at work has been supported as 
the single most influential work-related factor in the decision to attend work when ill (Hansen \& Andersen, 2008; Elstad \& Vabø, 2008; Aronsson \& Gustafsson, 2005). Finally, increased job demands also increase the likelihood of sickness presenteeism (Demerouti, Le Blanc, Bakker, Schaufeli \& Hox, 2009; Kivimaki et al., 2005) and lower performance (Van den Heuvel, Geuskens, Hooftman, Koppes \& Van den Bossche, 2010). Higher levels of presenteeism are associated with difficulties in staff replacement (Aronsson \& Gustafsson, 2005; Biron et al., 2006; Biron \& Saksvik, 2009; Dew et al., 2005; Jena, Baldwin, Daugherty, Meltzer \& Arora, 2010; Widera et al., 2010) and jobs that involve higher levels of teamwork (Hansen \& Andersen, 2008; Krohne \& Magnussen, 2011) and specifically higher interdependent work among small teams (Pauly, Nicholson, Polsky, Berger \& Sharda, 2008), and an increased responsibility at work (Dellve, Hadzibajramovic \& Ahlborg, 2011; Gosselin et al., 2013) that prompt employees to continue working when unwell. Employees who attended work whilst sick often do so because their colleagues are reliant on them and because they feel an obligation towards their team (Gosselin et al., 2013; McKevitt \& Morgan, 1997). Research into employment contract is inconclusive with some research showing that employees who have a permanent employment contract are more likely to come to work whilst ill than temporary staff (Aronsson, Gustafsson \& Dallner, 2000) and others showing no association between employment type and presenteeism behaviour (Aronsson \& Gustafsson, 2005; Hansen \& Andersen, 2008).

It should be noted that drawing conclusions on an individual phenomenon from national panel data (on which some these studies are based) may obscure some of the mechanisms of presenteeism that more fine-grained examinations could offer. Furthermore, correlational research highlights the wide range of work-related factors that can influence the decision to work while ill, but only allows to identify rather than explain causal mechanisms. For example, not if pressure to attend whilst sick is a potential mechanism, some of these 
factors can be viewed as salutogenic for performance and health (e.g., team cohesiveness, job satisfaction, engagement). Unfolding the psychological mechanisms by which these determinants lead to presenteeism behaviour can be helped by examining moderation and mediation mechanisms and reciprocal effects. For example, it has been shown that experience moderates the relationship between presenteeism and performance, such that more experienced nurses tend to be less affected by presenteeism as they completed their work more accurately (Martinez \& Ferreira, 2012).

Strongly implicated in presenteeism are also formal organizational policies and management practices, such as the existence of flexible work policies to create flexibility at work and adjust the work patterns of employees that were unwell so that they could have a more manageable workload (Krohne \& Magnussen, 2011), sick pay policies that allow employees paid sick days (Irvine, 2011; Chatterji \& Tilley, 2002; Heymann, Rho, Schmitt \& Earle, 2010), and better work organization or scheduling to allow for work reorganisation and unplanned absence (McKevitt \& Morgan, 1997).

Research also differentiates between formal organizational policies and workplace culture and climate. Salient differences between occupations in the incidences of presenteeism suggest that there may be variations in workplace cultures for presenteeism (Aronsson et al., 2000). A culture for presenteeism is grounded on employees' professional values as being responsible for vulnerable groups of people (Johns, 2010) as the overwhelming quantity of research on the prevalence of presenteeism in healthcare show (e.g., Crout, Chang \& Cioffi, 2005; Dew et al., 2005; Hackett \& Bycio, 1996; Karimi, Cheng, Bartram, Leggat \& Sarkeshik, 2015; Martinez \& Ferreira, 2012; Warren, White-Means, Wicks, Chang, Gourley \& Rice, 2011; Widera et al., 2010). Workplace norms that focus on responsibility, a strong work ethic, loyalty to team members, and attendance can also lead to presenteeism (McKevitt \& Morgan, 1997; Baker- McClearn et al., 2010; Dew et al., 2005; 
Simpson, 1998). Hansson, Boström and Harms-Ringdahl (2006) found that presenteeism levels were higher in organisations that expected employees to have strong work-duty norms even when they were ill (Dew et al., 2005; Vingård et al., 2004). There is also evidence that specific employee groups are more prone or high risk groups for presenteeism, highlighting more work group-specific circumstantial determinants of presenteeism. For example, higher levels of presenteeism have been detected among pregnant employees, whose fear of being considered as intellectually and physically inferior can lead them to exhibit higher levels of presenteeism and putting their health in danger (Gatrell, 2011), blue collar workers, who may be more self-conscious about their job security and an increased amount of "pressure to attend' than white-collar workers (Vroome, 2006), and nurses in a public hospital, who may experience a related reduction in performance increasing the number of errors and further impacting patient safety (Martinez \& Ferreira, 2012; Letvak, Ruhm \& Gupta, 2012). Finally, presenteeism poses challenges in $\boldsymbol{S M E s}$, which may experience its individual and economic consequences more acutely than larger organisations (Cocker et al., 2012; 2013). These challenges are primarily attributable to their size and structure, making administration, finance and responsibilities of human resource difficult (Rauch \& Frese, 2007).

Corroborating the available conceptual models, empirical research on singular antecedents shows that presenteeism behaviour is linked to an array of factors located at the individual, job, and organizational levels. Because presenteeism is highly responsive to the relationship between the individual and their work environment, it is an imperative to understand the interaction among these levels that influence presenteeism decisions and behaviour and the factors that moderate and mediate its effects on health and performance.

\section{Empirical research on the outcomes of presenteeism}

Optimal health is important for good performance and quality of working life, whereas poor health can lead to ill-health and counterproductive work behaviours. Next we 
outline empirical research on the range of negative as well as positive outcomes of presenteeism.

\section{Negative outcomes}

The volume of research on the negative outcomes of presenteeism is rich. In addition to financial costs (Hutchinson, 2011; Burton, Conti, Chen, Schultz \& Edington, 2002; LevinEpstein, 2005; CIPD, 2016; Cooper \& Dewe, 2008; Stewart et al., 2003a, 2003b), the costs of presenteeism for organizations tend to be ascribed to productivity loss (Goetzel, Hawkins, Ozminkowski \& Wang, 2003). Employees who are present at work when sick can experience subsequent declines in their overall performance (Biron et al., 2006; Cooper \& Dewe, 2008; Meerding, Ijzelenberg, Koopmanschap, Severens \& Burdorf, 2005; Van den Heuvel, Geuskens, Hooftman, Koppes \& Van den Bossche, 2010).

Despite the fact that presenteeism is viewed as responsible for decreased performance, there is surprisingly little empirical research on the relationship between the two. The available inconclusive research has highlighted a weak or non-existent relationship between presenteeism and performance (Johns, 2011; Munir, Jones, Leka \& Griffiths, 2005). Miraglia and Johns (2015) emphasise the role of the supervisor who may perceive presenteeism as something positive, encourage it, and consequently "reward it, assessing performance more positively, and this could nullify any negative relationship between presenteeism and rated job performance" (p. 14). More research on the dynamic relationship between presenteeism and performance is needed.

The relationships between presenteeism, on one hand, and physical ill-health and absenteeism, on the other, have also received substantial attention. Presenteeism can lead to a downward spiral of future health issues (Aronsson \& Gustafsson, 2005; Bergström et al., 2009; Kivimäki et al., 2005). For example, Kivimäki and his colleagues (2000) found an association between sickness presence and coronary heart disease and higher prevalence of 
absence leave. Furthermore, present and ill employees may spread their disease to others in the workplace, potentially leading to future sickness absenteeism among colleagues (Irvine, 2011; Widera et al., 2010). Presenteeism is also a risk factor for future poor health and sickness absence two years later, even after adjustment for possible confounders at baseline (Taloyan et al., 2012). Cross-sectional studies also link presenteeism with concurrent sickness absenteeism (Aronsson \& Gustafsson, 2005; Elstad and Vabo, 2008; Hansen \& Andersen, 2008; Leineweber et al., 2012). Brouwer, van Exel, Koopmanschap and Rutten (2002) showed that $35 \%$ of employees experienced presenteeism before or after absenteeism, also observed in Danish (Hansen \& Andersen, 2008), Nordic (Elstad \& Vabo, 2008), and Canadian employees (Caverley et al., 2007).

Prospective studies also show presenteeism to be a predictor of future sickness absenteeism (Bergström et al., 2009; Demerouti et al., 2009). Although working whilst sick may temporarily reduce rates of sickness absence recorded, higher future sickness absence levels are likely (De Vroome, 2006; Janssens, Clays, De Clercq, De Bacquer \& Braeckman, 2013). Using a follow-up period of 1.5 years, Hansen and Andersen (2009) revealed an association of sickness presence with long-term sickness absence of at least two weeks' duration and with spells lasting at least two months. Participants who had exhibited presenteeism more than six times in the pre-baseline year had a $74 \%$ higher risk of sickness absence for more than two months. Although the association was consistent for various symptoms and somatic conditions, it became weaker or insignificant for specific chronic conditions. In short, regular presenteeism is strongly linked to future long-term sickness absence (Hansen and Andersen, 2009), but this may depend on the specific health condition. Adjusting for previous sick leave and work-related variables, presenteeism becomes an important predictor of higher future sickness absenteeism (Bergström et al., 2009; Hansen \& Andersen, 2009; Kivimaki et al., 2005). It is important to consider the incidence and duration 
of sickness absence when examining the relationship between presenteeism and absenteeism. Janssens et al (2013) looked at different types of future sickness absence and found that high rates of presenteeism were associated with both long and short spells of sickness absence (of one to three days), moderate rates of presenteeism (two to five instances) were associated with long spells of sickness absence only for men, whereas high rates of presenteeism and high sickness frequency (at least three sick leave episodes) was demonstrated only among women.

Presenteeism has also been linked to low mental well-being and work ability (Gustafsson \& Marklund, 2011). Taloyan et al. (2012) attributed the association between presenteeism and suboptimal health largely to the mediating role of a higher risk of emotional exhaustion. Similarly, presenteeism has been linked to reduced job satisfaction via the mediating role of affective-motivational states such as work engagement and work addiction (Karanika-Murray et al., 2015). There is strong evidence that the relationship between sickness absence and presenteeism can be due to burnout incurred from individuals working beyond their physical or mental capabilities (De Vroome, 2006). Burnout increases sickness absence, which in turn increases the risk of subsequent presenteeism.

\section{Positive outcomes}

Although there is an implicit assumption that presenteeism is implicitly 'bad' and inevitably deleterious for health and performance, there are also suggestions that presenteeism may not always be taxing but rather that it can be beneficial for performance, well-being, and return to work, contradicting views that it is a risk factor for absenteeism (Bergström et al., 2009) and health (Bergström et al., 2009; Kivimäki, Head, Ferrie, Hemingway, Shipley \& Vahtera, 2005). Presenteeism can be beneficial for preventing accumulation of workload, gaining esteem from colleagues and managers (Vézina et al., 
2011) or achieving a sense of accomplishment, gradual recovery or citizenship behaviour (Miraglia \& Johns, 2015).

Presenteeism can reduce negative psychosocial effects of short or long-term absence from work. In cases where the health problem is benign, presenteeism can be used as an attempt for individuals to maintain their work performance during an illness (Demerouti et al., 2009). Employees who show up at work during illness may also feel more in control over their workload (Biron \& Saksvik, 2009). Furthermore, presenteeism may yield personal motivational benefits such as a sense of accomplishment that can help individuals adjust to work and cope with demands.

In terms of performance outcomes, working on less demanding tasks or with a lowered output can prevent the accumulation of work engendered by an absence, therefore potentially making the return to work less abrupt (Johns, 2008). As such, presenteeism may be a good strategy for maintaining well-being and facilitating recovery after long-term absence due to ill-health or injury (Ashby \& Mahdon, 2010). For example, Howard, Mayer and Gatchel (2009) found that the presenteeism group of chronic disabling musculoskeletal disorder patients who followed a functional restoration programme were more likely to return to full-time work one-year post-treatment, compared to the absentee group, and that presentees with chronic pain reported lower levels of depressive symptoms than absentees.

Presenteeism can also indirectly benefit teams and organizations because it can indicate commitment to colleagues and the organization, in turn create camaraderie within the workplace (Dew et al., 2005), impose less burden on colleagues who may otherwise be required to cover the absentee's work (Caverley et al., 2007), and generate approval from colleagues and managers (Biron \& Saksvik, 2009). In addition, presenteeism may also lead to reduced economic deprivation due to absence from work (Barnes, Buck, Williams, Webb \& Aylward, 2008). Nevertheless, it is unknown whether these effects are short-term; the 
findings do not preclude longer-term exhaustion and depersonalisation (see Demerouti et al., 2009), highlighting the possibility of concurrent positive and negative outcomes.

The range of positive outcomes of presenteeism supports the observation that, if well managed, presenteeism can be beneficial for longer term health and for maintaining performance and other desirable work outcomes (Karanika-Murray \& Biron, in preparation). As Miraglia and Johns (2015) write, "going to work while ill can represent a 'sustainable' choice" (p. 16). Occasions when presenteeism behaviour can have beneficial outcomes render the understanding of this "tipping point" (Biron \& Karanika-Murray, 2011) or "trade-off" (Johns, 2015) a worthwhile pursuit.

\section{Conclusions}

In this chapter we have examined the prevailing phenomenon of presenteeism, with the aim to unravel and decipher some of the major questions and issues in the field.

Deserving attention are aligning the measurement of presenteeism with accepted definitions, an understanding of how illness varieties and attendance options and how the two determine presenteeism behaviour, an examination of its outcomes and specifically potentially beneficial outcomes for health and performance, and most importantly the integration of research evidence and development of theory to explain why and how presenteeism happens. We hope to have inspired some lines of inspiring and rigorous research. This book is a move in the right direction for the field of presenteeism.

\section{References}

Admasachew, L., \& Dawson, J. (2011). The association between presenteeism and engagement of National Health Service staff. Journal of Health Services Research and Policy, 16(suppl 1), 29-33. 
Alavinia, S. M., Molenaar, D., \& Burdorf, A. (2009). Productivity loss in the workforce: associations with health, work demands, and individual characteristics. American Journal of Industrial Medicine, 52(1), 49-56.

Aronsson, G., \& Gustafsson, K. (2005). Sickness presenteeism: Prevalence, attendancepressure factors, and an outline of a model for research. Journal of Occupational and Environmental Medicine, 47(9), 958-966.

Aronsson, G., Gustafsson, K., \& Dallner, M. (2000). Sick but yet at work. an empirical study of sickness presenteeism. Journal of Epidemiology and Community Health, 54(7), 502-509.

Ashby, K., \& Mahdon, M. (2010). Why do employees come to work when ill? An investigation into sickness presence in the workplace Work Foundation.

Ashby, K., \& Mahdon, M. (2010). Why do employees come to work when ill: An investigation into sickness presence in the workplace. London: The Work Foundation.

Baker-McClearn, D., Greasley, K., Dale, J., \& Griffith, F. (2010). Absence management and presenteeism: The pressures on employees to attend work and the impact of attendance on performance. Human Resource Management Journal, 20(3), 311-328.

Barnes, M. C., Buck, R., Williams, G., Webb, K., \& Aylward, M. (2008). Beliefs about common health problems and work: A qualitative study. Social Science \& Medicine, 67(4), 657-665.

Bergström, G., Bodin, L., Hagberg, J., Aronsson, G., \& Josephson, M. (2009). Sickness Presenteeism Today, Sickness Absenteeism Tomorrow? A Prospective Study on Sickness Presenteeism and Future Sickness Absenteeism. Journal of Occupational \& Environmental Medicine, 51(6), 1-10.

Bierla, I., Huver, B., \& Richard, S. (2013). New evidence on absenteeism and presenteeism. International Journal of Human Resource Management, 24(7), 1536-1550. 
Biron, C., \& Karanika-Murray, M. (2011). Presenteeism and absenteeism: Critical points on a continuum. Paper presented at the 15th conference of the European Association of Work \& Organisational Psychology, 25-28 May, Maastricht.

Biron, C., \& Saksvik, P. Ø. (2009). Sickness presenteeism and attendance pressure factors: Implications for practice. International Handbook of Work and Health Psychology, 3.

Biron, C., Brun, J.-P., \& Ivers, H. (2006). At work but ill: Psychosocial work environment and wellbeing determinants of presenteeism propensity. Journal of Public Mental Health, 5(4), 26-37.

Brouwer, W. B., Van Exel, N., Koopmanschap, M., \& Rutten, F. (2002). Productivity costs before and after absence from work: As important as common? Health Policy, 61(2), 173-187.

Burton, W. N., Conti, D. J., Chen, C., Schultz, A. B., \& Edington, D. W. (1999). The role of health risk factors and disease on worker productivity. Journal of Occupational and Environmental Medicine, 41(10), 863-877.

Burton, W. N., Conti, D. J., Chen, C., Schultz, A. B., \& Edington, D. W. (2002). The economic burden of lost productivity due to migraine headache: A specific worksite analysis. Journal of Occupational and Environmental Medicine, 44(6), 523-529.

Burton, W. N., Pransky, G., Conti, D. J., Chen, C., \& Edington, D. W. (2004). The association of medical conditions and presenteeism. Journal of Occupational and Environmental Medicine, 46(6), S38-S45.

Caverley, N., Cunningham, J. B., \& MacGregor, J. N. (2007). Sickness presenteeism, sickness absenteeism, and health following restructuring in a public service organization. Journal of Management Studies, 44(2), 304-319.

Chapman, L. S. (2005). Presenteeism and its role in worksite health promotion. American Journal of Health Promotion, 19(4), 1-8. 
Chatterji, M., \& Tilley, C. J. (2002). Sickness, absenteeism, presenteeism, and sick pay. Oxford Economic Papers, 54(4), 669-687.

CIPD (2016). Absence management survey 2016. Available online at: https://www.cipd.co.uk/knowledge/fundamentals/relations/absence/absencemanagement-surveys

Cocker, F., Martin, A., Scott, J., Venn, A., \& Sanderson, K. (2012). Psychological distress and related work attendance among small-to-medium enterprise owner/managers: Literature review and research agenda. International Journal of Mental Health Promotion, 14(4), 219-236.

Cocker, F., Martin, A., Scott, J., Venn, A., \& Sanderson, K. (2013). Psychological distress, related work attendance, and productivity loss in small-to-medium enterprise owner/managers. International Journal of Environmental Research and Public Health, 10(10), 5062-5082.

Cooper, C. (1996). Hot under the Collar. Times Higher Education Supplement (June), 15.

Cooper, C., \& Dewe, P. (2008). Well-being--absenteeism, presenteeism, costs and challenges. Occupational Medicine, 58(8), 522-524.

Cooper, C., \& Lu, L. (2016). Presenteeism as a global phenomenon: Unraveling the psychosocial mechanisms from the perspective of social cognitive theory. Cross Cultural \& Strategic Management, 23(2), 216-231.

Crout, L. A., Chang, E., \& Cioffi, J. (2005). Why do registered nurses work when ill?. Journal of Nursing Administration, 35(1), 23-28.

Cyr, A., \& Hagen, S. (2007). Measurement and quantification of presenteeism. Journal of Occupational and Environmental Medicine, 49(12), 1299-1300. 
De Vries, H. J., Brouwer, S., Groothoff, J. W., Geertzen, J. H., \& Reneman, M. F. (2011). Staying at work with chronic nonspecific musculoskeletal pain: a qualitative study of workers' experiences. BMC Musculoskeletal Disorders, 12(1), 126.

De Vroome, E. D. (2006). Prevalence of sickness absence and 'presenteeism'. European Foundation for the Improvement of Living and Working Conditions (Eurofound). Available online at: https://www.eurofound.europa.eu/observatories/eurwork/articles/workingconditions/prevalence-ofsickness-absence-and-presenteeism

Dellve, L., Hadzibajramovic, E., \& Ahlborg Jr, G. (2011). Work attendance among healthcare workers: prevalence, incentives, and long- term consequences for health and performance. Journal of Advanced Nursing, 67(9), 1918-1929.

Demerouti, E., Le Blanc, P. M., Bakker, A. B., Schaufeli, W. B., \& Hox, J. (2009). Present but sick: A three-wave study on job demands, presenteeism and burnout. Career Development International, 14(1), 50-68.

Dew, K., Keefe, V., \& Small, K. (2005). Choosing to work when sick: workplace presenteeism. Social Science and Medicine, 60, 2273-2282.

Dickson, V.V. (2013). Presenteeism among older workers ( $\geq 45$ years) with coronary heart disease: an integrative literature review. Ohio Public Health Journal, 6, 31-41.

Elstad, J. I., \& Vabo, M. (2008). Job stress, sickness absence and sickness presenteeism in nordic elderly care. Scandinavian Journal of Public Health, 36(5), 467-474.

Garrow, V. (2016). Presenteeism: A review of current thinking. Institute for Employment Studies Report 507.

Gatrell, C. J. (2011). 'I'ma bad mum': Pregnant presenteeism and poor health at work. Social Science and Medicine, 72(4), 478-485. 
Goetzel, R. Z., Hawkins, K., Ozminkowski, R. J., \& Wang, S. (2003). The health and productivity cost burden of the "top 10" physical and mental health conditions affecting six large US employers in 1999. Journal of Occupational and Environmental Medicine, 45(1), 5-14.

Gosselin, E., Lemyre, L., \& Corneil, W. (2013). Presenteeism and absenteeism: Differentiated understanding of related phenomena. Journal of Occupational Health Psychology, 18(1), 75-86.

Grinyer, A., \& Singleton, V. (2000). Sickness absence as risk-taking behavior: A study of organisational and cultural factors in the public sector. Health, Risk \& Society, 2(1), $7-21$.

Gustafsson, K., \& Marklund, S. (2011). Consequences of sickness presence and sickness absence on health and work ability: A swedish prospective cohort study. International Journal of Occupational Medicine and Environmental Health, 24(2), 153-165.

Hackett, R. D., \& Bycio, P. (1996). An evaluation of employee absenteeism as a coping mechanism among hospital nurses. Journal of Occupational and Organizational Psychology, 69(4), 327-338.

Halbesleben, J. R., Whitman, M. V., \& Crawford, W. S. (2014). A dialectical theory of the decision to go to work: Bringing together absenteeism and presenteeism. Human Resource Management Review, 24(2), 177-192.

Hansen, C. D., \& Andersen, J. H. (2008). Going ill to work-What personal circumstances, attitudes and work-related factors are associated with sickness presenteeism? Social Science \& Medicine, 67(6), 956-964.

Hansen, C. D., \& Andersen, J. H. (2009). Sick at work--a risk factor for long-term sickness absence at a later date? Journal of Epidemiology and Community Health, 63(5), 397402. 
Hansson, M., Boström, C., \& Harms-Ringdahl, K. (2006). Sickness absence and sickness attendance — what people with neck or back pain think. Social Science and Medicine, 62(9), 2183-2195.

Hemp, P. (2004). Presenteeism: At work-but out of it. Harvard Business Review, 82(10), 4958.

Heymann, J., Rho, H. J., Schmitt, J., \& Earle, A. (2010). Ensuring a healthy and productive workforce: Comparing the generosity of paid sick day and sick leave policies in 22 countries. International Journal of Health Services, 40(1), 1-22.

Howard, K. J., Mayer, T. G., \& Gatchel, R. J. (2009). Effects of presenteeism in chronic occupational musculoskeletal disorders: Stay at work is validated. Journal of Occupational and Environmental Medicine, 51(6), 724-731.

Hutchinson, J. (2011, Sainsbury Centre for Mental Health. Presenteeism. Retrieved from www.centreformentalhealth.org.uk/training/why_working_late.aspx

Irvine, A. (2011). Fit for work? the influence of sick pay and job flexibility on sickness absence and implications for presenteeism. Social Policy \& Administration, 45(7), $752-769$.

Janssens, H., Clays, E., De Clercq, B., De Bacquer, D., \& Braeckman, L. (2013). The relation between presenteeism and different types of future sickness absence. Journal of Occupational Health, 55(3), 132-141.

Jena, A. B., Baldwin, D. C., Daugherty, S. R., Meltzer, D. O., \& Arora, V. M. (2010). Presenteeism among resident physicians. JAMA, 304(11), 1166-1168.

Johansson, G., \& Lundberg, I. (2004). Adjustment latitude and attendance requirements as determinants of sickness absence or attendance. empirical tests of the illness flexibility model. Social Science \& Medicine, 58(10), 1857-1868. 
Johns, G. (2008). Absenteeism or presenteeism? attendance dynamics and employee wellbeing. In S. Cartwright, \& C. L. Cooper (Eds.), The oxford handbook of organizational well-being (pp. 7-30). Oxford: Oxford University Press.

Johns, G. (2010). Presenteeism in the workplace: A review and research agenda. Journal of Organizational Behavior, 31(4), 519-542.

Johns, G. (2011). Attendance dynamics at work: The antecedents and correlates of presenteeism, absenteeism, and productivity loss. Journal of Occupational Health Psychology, 16(4), 483.

Karanika-Murray, M., \& Biron, C. (in preparation). Functional presenteeism? Can presenteeism be a sustainable choice for well -being and performance?

Karanika-Murray, M., Pontes, H. M., Griffiths, M. D., \& Biron, C. (2015). Sickness presenteeism determines job satisfaction via affective-motivational states. Social Science and Medicine, 139, 100-106.

Karimi, L., Cheng, C., Bartram, T., Leggat, S. G., \& Sarkeshik, S. (2015). The effects of emotional intelligence and stress- related presenteeism on nurses' well- being. Asia Pacific Journal of Human Resources, 53(3), 296-310.

Kivimäki, M., Head, J., Ferrie, J. E., Hemingway, H., Shipley, M. J., Vahtera, J., \& Marmot, M. G. (2005). Working while ill as a risk factor for serious coronary events: The whitehall II study. American Journal of Public Health, 95(1), 98-102.

Kivimäki, M., Vahtera, J., Pentti, J., \& Ferrie, J. E. (2000). Factors underlying the effect of organisational downsizing on health of employees: Longitudinal cohort study. British Medical Journal (Clinical Research Education), 320(7240), 971-975.

Krohne, K., \& Magnussen, L. H. (2011). Go to work or report sick? A focus group study on decisions of sickness presence among offshore catering section workers. BMC Research Notes, 4, 70-0500-4-70. 
Leineweber, C., Westerlund, H., Hagberg, J., Svedberg, P., \& Alexanderson, K. (2012). Sickness presenteeism is more than an alternative to sickness absence: Results from the population-based SLOSH study. International Archives of Occupational and Environmental Health, 85(8), 905-914.

Levin-Epstein, J. (2005). Presenteeism and Paid Sick Days. Available online at: http://www.issuelab.org/click/download1/presenteeism_and_paid_sick_days/clasp.org /publications/presenteeism.pdf

Loeppke, R., Taitel, M., Haufle, V., Parry, T., Kessler, R. C., \& Jinnett, K. (2009). Health and productivity as a business strategy: a multiemployer study. Journal of Occupational and Environmental Medicine, 51(4), 411-428.

Martinez, L. F., \& Ferreira, A. I. (2012). Sick at work: presenteeism among nurses in a Portuguese public hospital. Stress and Health, 28(4), 297-304.

McKevitt, C., \& Morgan, M. (1997). Illness doesn't belong to us. Journal of the Royal Society of Medicine, 90(9), 491-495.

McTernan, W. P., Dollard, M. F., \& LaMontagne, A. D. (2013). Depression in the workplace: An economic cost analysis of depression-related productivity loss attributable to job strain and bullying. Work \& Stress, 27(4), 321-338.

Meerding, W., IJzelenberg, W., Koopmanschap, M., Severens, J. L., \& Burdorf, A. (2005). Health problems lead to considerable productivity loss at work among workers with high physical load jobs. Journal of Clinical Epidemiology, 58(5), 517-523.

Mellers, B. A., Schwartz, A., \& Cooke, A. D. J. (1998). Judgement and decision making. Annual Review of Psychology, 49, 447-477.

Miraglia, M., \& Johns, G. (2016). Going to work ill: A meta-analysis of the correlates of presenteeism and a dual-path model. Journal of Occupational Health Psychology, 21(3), 261. 
Morgeson, F. P., \& Hofmann, D. A. (1999). The structure and function of collective constructs: Implications for multilevel research and theory development. Academy of Management Review, 24(2), 249-265.

Munir, F., Jones, D., Leka, S., \& Griffiths, A. (2005). Work limitations and employer adjustments for employees with chronic illness. International Journal of Rehabilitation Research, 28(2), 111-117.

Munir, F., Yarker, J., Haslam, C., Long, H., Leka, S., Griffiths, A., \& Cox, S. (2007). Work factors related to psychological and health-related distress among employees with chronic illnesses. Journal of Occupational Rehabilitation, 17(2), 259-277.

Pauly, M. V., Nicholson, S., Polsky, D., Berger, M. L., \& Sharda, C. (2008). Valuing reductions in on- the- job illness: 'presenteeism' from managerial and economic perspectives. Health Economics, 17(4), 469-485.

Prater, T., \& Smith, K. (2011). Underlying factors contributing to presenteeism and absenteeism. Journal of Business \& Economics Research, 9(6), 1-14.

Rauch, A., \& Frese, M. (2007). Let's put the person back into entrepreneurship research: A meta-analysis on the relationship between business owners' personality traits, business creation, and success. European Journal of Work and Organizational Psychology, $16(4), 353-385$.

Ridic, G., Gleason, S., \& Ridic, O. (2012). Comparisons of health care systems in the United States, Germany and Canada. Materia Socio-Medica, 24(2), 112.

Rosvold, E.O., \& Bjertness, E. (2001). Physicians who do not take sick leave: hazardous heroes? Scandinavian Journal of Public Health, 29(1), 71 - 75

Schultz, A. B., \& Edington, D. W. (2007). Employee health and presenteeism: A systematic review. Journal of Occupational Rehabilitation, 17(3), 547-579. 
Simpson, R. (1998). Presenteeism, power and organizational change: Long hours as a career barrier and the impact on the working lives of women managers. British Journal of Management, 9(s1), 37-50.

St-Arnaud, L. (2007). Determinants of return-to-work among employees absent due to mental health problems. Industrial Relations, 62(4), 690.

Stewart, W. F., Ricci, J. A., Chee, E., Hahn, S. R., \& Morganstein, D. (2003a). Cost of lost productive work time among US workers with depression. Jama, 289(23), 3135-3144.

Stewart, W. F., Ricci, J. A., Chee, E., Morganstein, D., \& Lipton, R. (2003b). Lost productive time and cost due to common pain conditions in the US workforce. Jama, 290(18), $2443-2454$.

Taloyan, M., Aronsson, G., Leineweber, C., Hanson, L. M., Alexanderson, K., \& Westerlund, H. (2012). Sickness presenteeism predicts suboptimal self-rated health and sickness absence: A nationally representative study of the Swedish working population. PLoS One, 7(9), e44721.

Van den Heuvel, S. G., Geuskens, G. A., Hooftman, W. E., Koppes, L. L., \& Van den Bossche, S. N. (2010). Productivity loss at work; health-related and work-related factors. Journal of Occupational Rehabilitation, 20(3), 331-339.

Vézina, M., Cloutier, E., Stock, S., Lippel, K., Fortin, É, Delisle, A., . . Prud'homme, P. (2011). Enquête québécoise sur des conditions de travail, d'emploi et de SST (EQCOTESST). ( No. R-691). Montréal: IRSST.

Vingård, E., Alexanderson, K., \& Norlund, A. (2004). . Sickness presence. Scandinavian Journal of Public Health, 32(63 suppl), 216-221.

Vroome, E. D. (2006). Prevalence of sickness absence and presenteeism. European Foundation for the Improvement of Living and Working Conditions. Available online 
at: https://www.eurofound.europa.eu/observatories/eurwork/articles/workingconditions/prevalence-of-sickness-absence-and-presenteeism

Warren, C. L., White-Means, S. I., Wicks, M. N., Chang, C. F., Gourley, D., \& Rice, M. (2011). Cost burden of the presenteeism health outcome: diverse workforce of nurses and pharmacists. Journal of Occupational and Environmental Medicine, 53(1), 90-99.

Widera, E., Chang, A., \& Chen, H. L. (2010). Presenteeism: A public health hazard. Journal of General Internal Medicine, 25(11), 1244-1247. 